\title{
Quality of Life Outcomes after Functional Endoscopic Sinus Surgery
}

\author{
Zachary M. Soler, MD and Timothy L. Smith, MD, MPH \\ Division of Rhinology and Sinus Surgery, Oregon Sinus Center Department of Otolaryngology - \\ Head and Neck Surgery Oregon Health and Science University Portland, Oregon, USA
}

\begin{abstract}
Most patients with chronic rhinosinusitis seek medical treatment when the burden of symptoms negatively impacts their quality of life. The degree to which quality of life improves after sinus surgery is thus a critical indicator of surgical success. This article reviews quality of life outcomes after functional endoscopic sinus surgery, including relevant clinical factors, weaknesses in the current literature, and future research directions.
\end{abstract}

\section{Keywords}

Chronic Rhinosinusitis; Quality of life; Endoscopic sinus surgery; Sinusitis

\section{Introduction}

Clinicians have traditionally focused on objective findings in order to assess response after a given treatment; however, for many disease processes including chronic rhinosinusitis (CRS), objective measures fail to capture the full burden of disease experienced by the individual patient. The discordance between radiographic findings and patients' symptoms in CRS highlights this dilemma. Numerous studies have shown that the degree of sinonasal inflammation as measured by computed tomography (CT) scan or endoscopy fails to correlate with the extent of symptoms experienced by the individual patient ${ }^{1}$. A patient may therefore have debilitating symptoms with only minimal mucosal thickening or vice versa. The lack of agreement between objective assessment and patient-centered assessment is not unique to CRS, but can also be seen in conditions such as obstructive sleep apnea, asthma, and low back pain $^{2-5}$. At present, CRS remains a symptom-based diagnosis (corroborated by objective signs of inflammation) and the extent of symptoms remains the overriding factor motivating patients to seek medical treatment ${ }^{6}$. Given this situation, the study of patient-centered disease impact is critical to understanding outcomes after endoscopic sinus surgery (ESS). To date, a number of rhinologic-specific instruments have been developed to measure quality-of-life (QOL) in patients with rhinologic conditions, including the CSS, RSDI, RQLQ, and most recently the

(C) 2010 Elsevier Inc. All rights reserved.

Corresponding Author: Timothy L. Smith, MD, MPH Division of Rhinology and Sinus Surgery Department of Otolaryngology - Head and Neck Surgery Oregon Health and Science University 3181 SW Sam Jackson Park Rd PV-01 Portland, OR 97239 503-494-4631 fax smithtim@ohsu.edu.

Publisher's Disclaimer: This is a PDF file of an unedited manuscript that has been accepted for publication. As a service to our customers we are providing this early version of the manuscript. The manuscript will undergo copyediting, typesetting, and review of the resulting proof before it is published in its final citable form. Please note that during the production process errors may be discovered which could affect the content, and all legal disclaimers that apply to the journal pertain. 
SNOT-22 $2-10$. These instruments provide a validated means to objectively quantify a patient's perception of their disease burden both before and after intervention.

\section{In General, QOL Improves After ESS}

In general, patient-reported symptoms and QOL improve after ESS. One of the earliest studies was published by Kennedy in 1992, wherein $97 \%$ of 120 patients reported improvement in symptoms (85\% marked improvement) after ESS with a mean follow-up of 18 months ${ }^{11}$. A follow-up study on the same cohort showed these results to be durable up to 7.8 years after surgery ${ }^{12}$. The development of validated, disease-specific QOL instruments in the mid 1990s added an additional layer of sophistication to ESS outcomes research. A recent literature review identified 45 articles published between 1966 and 2004 dedicated to the question of whether ESS leads to improvements in symptoms and/or QOL ${ }^{13}$. In that report, all 45 studies demonstrated improvements in CRS-related symptoms and QOL, including 11 prospective studies and 5 which utilized validated QOL instruments.

Since that time, additional large prospective studies have been published utilizing validated CRS-specific QOL instruments or symptom scales. Kountakis followed a cohort of 158 patients for 12 months after ESS, reporting statistically significant improvements in patient visual analog scale (VAS) scores for Rhinosinusitis Task Force (RSTF) symptoms ${ }^{14}$. Major RSTF symptom scores ranged from 4.5-5.7 (0-10 point scale) at baseline and improved to 0.3 to 0.9 after ESS, representing a greater than $80 \%$ change from baseline. SNOT-20 scores were also shown to improve by $77 \%$ after surgery.

Bhattacharyya performed a similar study following 100 patients for an average of 19 months after ESS $^{15}$. RSI symptoms were examined at preoperative and postoperative time points utilizing Likert scales (0-5 range). After surgery, statistically significant decreases in major (facial pressure, nasal congestion, nasal obstruction, rhinorrhea, and hyposmia) and minor symptoms were noted $(\mathrm{p}<0.001$ for all). The net change in major symptom score ranged from 1.5 to 2.3 points ( $0-5$ range) with effect sizes noted to be large.

Recent studies have also focused on nonspecific symptoms such as fatigue and bodily pain. Chester performed a systematic review and meta-analysis of available studies looking at the effect of ESS on fatigue, vitality, energy and malaise ${ }^{16}$. All 28 identified studies described substantial improvement in fatigue following ESS. A similar study by the same author showed improvements in bodily pain scores as recorded by the SF-36 instrument ${ }^{17}$.

One of the most comprehensive studies was published by Smith et al in $2009^{18}$. This multiinstitutional study prospectively followed 302 patients at three medical centers for an average of 17.4 months after ESS. Mean scores improved on the RSDI by 18.9 points $(15.8 \%$; $\mathrm{p}<0.001)$ and the CSS by 21.2 points $(21.2 \%$; $\mathrm{p}<0.001)$. Put another way, among patients with poor baseline QOL, 71.7\% experienced clinically significant improvement on the RSDI and 76.1\% on the CSS.

The available literature provides a wealth of evidence demonstrating that CRS-specific symptoms and QOL improve after ESS to statistically significant levels; however, a "statistically significant" improvement may not necessarily translate into a clinically relevant change as perceived by the individual patient. One of the burdens of QOL research is to identify what has been called the "minimal clinically important difference" (MCID). The MCID is the minimal change in symptom or QOL after a given intervention which is perceptible and thus relevant to the individual patient. Establishing an MCID for a particular disease state and QOL instrument can be a difficult task. Until recently, little had been done to specifically define the MCID for CRS symptoms or CRS-specific QOL instruments. Instead, estimates of MCID were extrapolated from other disease states and used by proxy. For example, there has been much 
interest using VAS scales for measuring pain in the operative and emergency department setting. Prior studies have shown the MCID for acute pain to be between 0.9 to 1.3 (10 point scale $)^{19-21}$. Recent studies reporting scaled symptom scores by Kountakis, Bhattacharrya, and Smith all easily reach this threshold of clinical relevance ${ }^{14,15,22}$. Similarly, estimates of MCID exist for general QOL instruments such as the SF-36, wherein 10 to 12.5 points (100 point scale) represents the minimum change felt to be clinically relevant for diseases such as asthma, chronic obstructive pulmonary disease and coronary artery disease ${ }^{23}$. Studies by Smith and Kennedy evaluating long-term changes in SF-36 scores easily exceed these extrapolated thresholds ${ }^{11,18}$.

The difficulty of defining an MCID is not unique to rhinologic QOL outcomes research. The most widely accepted solution in other disease states has been to use statistical constructs such as standard error and standard deviation to define the MCID. Generally speaking, QOL changes become clinically meaningful when they approximate $1 / 2$ of the standard deviation of the baseline QOL value for the given population. This seemingly arbitrary definition of clinical relevance has been validated across many disease-specific and general QOL instruments ${ }^{24}$. Considering the multi-institutional outcomes study by Smith et al, this threshold of clinical significance was exceeded for both the disease-specific (CSS, RSDI) and general QOL improvements after ESS ${ }^{18}$. Recently, attention has been given to defining the MCID for CRSspecific QOL instruments including the RQLQ and SNOT-22. Table 1 shows the available published MCID values for CRS-specific QOL instruments ${ }^{10,18,25}$.

Even with the beneficial outcomes seen after ESS, one must not confuse "improvement" with complete symptomatic resolution or "cure." ESS is most typically reserved for patients who are refractory to standard medical treatments. The preponderance of the QOL studies show that these patients will experience statistically and clinically significant improvement after ESS, but will likely still be left with some measurable burden of disease. For example, patients in the Bhattacharyya study showed average postoperative symptom scores ranging from 1.0 to $1.4(0-5 \text { scale })^{15}$. Table 2 shows symptom scores from a different cohort both before and after endoscopic sinus surgery ${ }^{22}$. Similarly, the multi-institutional Smith study reported average postoperative RSDI and CSS scores well above the norms for those without CRS ${ }^{18}$. These studies were done at tertiary referral centers and usually include a disproportionate number of patients with severe phenotype and revision surgical status. As such, reported results might not be fully generalizable to all patients undergoing ESS.

\section{Various Clinical Factors Can Influence QOL}

As detailed above, the majority of patients will perceive improvement in symptoms and QOL after ESS. However, there is variability as to the degree with which any individual patient will improve. Much attention has been given to identifying clinical factors which might enable treating physicians to predict a favorable or unfavorable outcome. Studies have looked at demographic factors (age, gender, ethnicity), medical comorbidities (asthma, allergies, ASA triad disease, depression, fibromyalgia, smoking, prior sinus surgery), and phenotypic qualities (polyposis, eosinophils) in order to identify characteristics which might allow outcome prediction ${ }^{18,26-34}$. The results of these studies can at times seem conflicting given the subtle but important differences in study design/analysis as well as the interrelatedness of various factors which makes confounding difficult to fully eliminate.

Several factors have been shown to worsen baseline sinusitis-specific QOL, including ASA triad disease, depression, fibromyalgia, female gender, and non-Caucasian ethnicity 18,27,29, 33. Patients with polyps and those of male gender have been shown to have slightly higher baseline QOL than the average CRS patient ${ }^{26,33}$. Asthma, allergies, age, and revision surgical status seem to have little or no affect on baseline $\mathrm{QOL}^{18,28,31,32,33}$. Regardless of their affect 
on baseline disease-specific QOL, few of the above factors seem to influence the degree of improvement afforded by ESS. For example, patients with comorbid depression have worse disease-specific QOL scores at baseline and postoperatively compared to those without depression. However, they will experience the same absolute level of improvement with ESS as those without depression ${ }^{27}$. Thus, at any one point in time a depressed patient will have worse QOL than a non-depressed patient, but the absolute change in QOL after ESS will be similar to those without depression. Similar results have been seen when closely examining patients with ASA triad disease, fibromyalgia, female gender, and non-Caucasian ethnicities $18,29,30,33$. One recent study reported that a history of prior sinus surgery predicted less improvement in QOL after ESS ${ }^{18}$. In that study, patients undergoing primary ESS were 2.1 times more likely to improve as patients undergoing revision surgery (95\% CI 1.2 to 3.4; $\mathrm{p}<0.006)$. No other factor was found to be similarly predictive by multivariate regression analysis.

Patients with nasal polyposis represent a unique clinical subgroup. The presence of nasal polyps naturally leads to worse objective disease severity as defined by imaging and endoscopic grading systems ${ }^{15}$. However, except for symptoms of nasal congestion, these patients appear to have a lighter burden of disease as measured by patient-centered symptom scores and QOL compared to patients without nasal polyps ${ }^{26}$. Most clinicians have met the archetypal patient with polyps completely filling the nasal cavity yet relatively few complaints. Despite better preoperative QOL scores, patients with polyps tend to improve after ESS to a similar degree as those without polyps.

Recently, we reported the impact of mucosal eosinophilia on QOL outcomes in 102 patients with $\mathrm{CRS}^{34}$. At the time of ESS, the degree of mucosal eosinophilia was quantified via microscopy. Although all patients demonstrated improvement after ESS, the subgroup of patients with mucosal eosinophilia (greater than 10 eosinophils/high powered field (HPF) showed less improvement in disease-specific (CSS, RSDI) and general (SF-36) QOL than those without eosinophilia (less than 10 eosinophils/HPF) at an average follow-up of 16 months. Interestingly, patients without polyps but with mucosal eosinophilia performed the worst after ESS and patients without polyps or eosinophilia performed the best. The difference in outcomes between the groups clearly exceeded a threshold one would consider clinically relevant. Without pathologic review, these patients might otherwise be indistinguishable on clinical grounds alone. These findings are not unexpected and serve to support the commonly held clinical belief that patients with eosinophilic inflammation are especially difficult to treat.

\section{Important Weaknesses Exist in Current QOL Outcomes Studies}

Outcomes research for CRS has steadily improved over the last 25 years from short-term, single-institution studies of retrospective nature to prospective, multi-institution studies utilizing validated disease-specific QOL instruments. Despite these improvements, important weaknesses still exist in the current literature. The lack of a control arm is perhaps the biggest shortcoming of most studies. A control arm serves to minimize the chance that observed improvements simply represent the natural resolution of the disease process. Additionally, a control arm would minimize the placebo affect associated with any given treatment.

Randomized, double-blind, placebo-controlled trials (RDBPCT) remain the gold standard for many disease processes (level 1 evidence) and serve as the foundation for evidence-based reviews such as The Cochrane Database. However, the tenants of a RDBPCT are not easily applied to surgical outcomes as obvious problems with randomization, blinding, and sham surgeries arise. To our knowledge, only two randomized studies have compared medical regimens to ESS for adult patients with $\mathrm{CRS}^{35,36}$. Both of these were done outside of the United States and showed no difference in outcomes when comparing ESS to medical treatments of 
CRS. The Cochrane Review of these trials thus concluded that available evidence does not demonstrate ESS conferring additional benefit over medical treatment alone ${ }^{37}$. However, the impact of the Cochrane Review has been mitigated by the fact that patients were randomized to ESS prior to receiving maximal medical treatments. The comparison was thus ESS versus a medical regimen as initial treatment of CRS. This up-front, direct comparison between surgery and medical therapy does not justly model current typical practice patterns. The typical current paradigm is to first treat CRS patients with maximal available medical treatments which often include antibiotic and anti-inflammatory regimens. Only those who fail these interventions are offered ESS, usually followed by ongoing medical treatments. Thus, to best mimic current treatment paradigms, one would begin with a cohort that has failed maximal medical management, and compare further treatment with ESS plus ongoing medical management to a control arm of continued medical management (level 2 evidence). Studies with the above construct would represent the strongest evidence likely to be achieved in the United States.

\section{Future Outcomes Studies Should Incorporate Histologic, Molecular and Genetic Information}

The initial objective of ESS outcomes research was to assess the effectiveness of ESS as a therapeutic option for CRS in general. Current thinking is that CRS represents a generic diagnosis of which there are many underlying etiologies and phenotypic subtypes. Various classifications schemes exist often based on clinical features such as presence of polyps and/ or mucosal eosinophilia, although little consensus exists. As subtypes of chronic rhinosinusitis are identified and precisely classified, it will be important to understand variations in outcomes related to available therapies. Perhaps the greatest goal is to develop a comprehensive model of multiple pretreatment factors which predict disease-specific QOL outcomes after treatment. This model would include demographic, clinical, and radiographic measures as previously discussed. The discovery that mucosal eosinophilia predicts QOL outcomes after ESS suggests that further investigation is needed to define the underlying basis and characteristics of the inflammatory infiltrate comprising CRS. Evaluating cellular counts and other histologic parameters of mucosal inflammation represents a fairly crude method of assessment as compared with current molecular and genetic methodology. The study of molecular markers of inflammation and their genetic underpinnings remains a nascent research front just now beginning to flourish. Future studies will need to incorporate genetic markers and molecular markers of genetic expression into predictive models before a clear, comprehensive picture of outcomes is finally realized.

\section{Conclusions}

\section{Implications for Clinical Practice}

An abundance of evidence exists supporting the efficacy of ESS to improve long-term QOL outcomes in patients with CRS. Both CRS-specific and general QOL improve after ESS to levels considered statistically significant and clinically relevant. Variability in individual patient QOL can in part be explained by demographic factors, medical comorbidities, and histologic inflammatory phenotypes.

\section{Implications for Research}

Continued QOL outcomes research is necessary in both the medical and surgical treatment of CRS. Control arms should be incorporated where possible to minimize confounding and placebo effects. An overarching goal is to develop a comprehensive model of multiple pretreatment factors which predicts QOL outcomes and would guide clinical decision making. 
This model will likely need to incorporate clinical, histologic, molecular, and genetic information to fully account for individual variability.

\section{References}

1. Stewart MG, Smith TL. Objective versus subjective outcomes assessment in rhinology. Am J rhinol 2005;19:529-535. [PubMed: 16270610]

2. Weaver EM, Kapur V, Yueh B. Polysomnography vs self-reported measures in patients with sleep apnea. Arch Otolaryngol Head Neck Surg 2004;130:453-458. [PubMed: 15096430]

3. Kemp JP, Cook DA, Incaudo GA, et al. Salmeterol improves quality of life in patients with asthma requiring inhaled corticosteroids. J Allergy Clin Immunol 1998;101:188-195. [PubMed: 9500751]

4. Fuchs-Clement D, Le Gallais D, Varray A, et al. Factor analysis of quality of life in patients with chronic obstructive pulmonary disease before and after rehabilitation. Am J Phys Med Rehabil 2001;80:113-120. [PubMed: 11212011]

5. Gronblad M, Hurri H, Kouri JP. Relationships between spine mobility, physical performance tests, pain intensity, and disability assessments in chronic low back pain patients. Scand J Rehab Med 1997;29:17-24.

6. Rosenfeld RM, Andes D, Bhattacharyya N, et al. Clinical practice guidelines: adult sinusitis. Otolaryngol Head Neck Surg 2007;137(Supplement):S1-S31. [PubMed: 17761281]

7. Leopold D, Ferguson BJ, Piccirillo JF. Outcomes assessment. Otolaryngol Head Neck Surg 1997;117 (Supplement):S58-S68. [PubMed: 9334790]

8. Gliklich RE, Metson R. Techniques for outcomes research in surgery for chronic sinusitis. Laryngoscope 1995;105:387-390. [PubMed: 7715384]

9. Benninger MS, Senior BA. The development of the rhinosinusitis disability index. Arch Otolaryngol Head Neck Surg 1997;123:1175-1179. [PubMed: 9366696]

10. Hopkins C, Gillett S, Slack R, Lund VJ, Browne JP. Psychometric validity of the 22-item Sinonasal Outcome Test. Clin Otol 2009;34:447-454.

11. Kennedy DW. Prognostic factors, outcomes, and staging in ethmoid sinus surgery. Laryngoscope 1992;102:1-18. [PubMed: 1453856]

12. Senior BA, Kennedy DW, Tanabodee J, et al. Long-term results of functional endoscopic sinus surgery. Laryngoscope 1998;108:151-157. [PubMed: 9473061]

13. Smith TL, Batra PS, Seiden AM, et al. Evidence supporting endoscopic sinus surgery in the management of adult chronic rhinosinusitis: a systematic review. Am J Rhinol 2005;19:537-543. [PubMed: 16402637]

14. Ling FT, Kountakis SE. Important clinical symptoms in patients undergoing functional endoscopic sinus surgery for chronic rhinosinusitis. Laryngoscope 2007;117:1090-1093. [PubMed: 17440425]

15. Bhattacharyya N. Symptom outcomes after endoscopic sinus surgery for chronic rhinosinusitis. Arch Otolaryngol Head Neck Surg 2005;130:329-333. [PubMed: 15023842]

16. Chester AC, Sindwani R, Smith TL, Bhattacharyya N. Fatigue improvement following endoscopic sinus surgery: a systematic review and meta-analysis. Laryngoscope 2008;118:730-739. [PubMed: 18216743]

17. Chester AC, Sindwani R, Smith TL, Bhattacharyya N. Systematic review of change in bodily pain after sinus surgery. Otolaryngol Head Neck Surg 2008;139:759-765. [PubMed: 19041499]

18. Smith TL, Litvack JR, Hwang PW, et al. Determinants of outcomes of sinus surgery: a multiinstitutional prospective cohort study. Otolaryngol Head Neck Surg 2010;142:55-63. [PubMed: 20096224]

19. Powell CV, Kelly AM, Williams A. Determining the minimum clinically significant difference in visual analog pain score for children. Ann Emerg Med 2001;37:28-31. [PubMed: 11145767]

20. Todd KH, Funk KG, Funk JP, Bonacci R. Clinical significance of reported changes in pain severity. Ann Emerg Med 1996;27:485-489. [PubMed: 8604867]

21. Kelly AM. Does the clinically significant difference in visual analog scale pain scores vary with gender, age, or cause of pain? Acad Emerg Med 1998;5:1086-1090. [PubMed: 9835471] 
22. Soler ZM, Mace J, Smith TL. Symptom-based presentation of chronic rhinosinusitis and symptomspecific outcomes after endoscopic sinus surgery. Am J Rhinol 2008;22:297-301. [PubMed: $18588763]$

23. Wyrwick KW, Tierney WM, Babu AN, et al. A comparison of clinically important differences in health-related quality of life for patients with chronic lung disease, asthma, or heart disease. Health Serv Res 2005;40(2):577-592. [PubMed: 15762908]

24. Norman GR, Sloan JA, Wyrwick KW. Interpretation of changes in health related quality of life: the remarkable universality of half a standard deviation. Med Care 2003;41:582-592. [PubMed: 12719681]

25. Turner D, Schunemann HJ, Griffith LE, et al. Using the entire cohort in the receiver operating characteristic analysis maximizes precision of the minimal important difference. J Clin Epidemiol 2009;62:374-379. [PubMed: 19013766]

26. Poetker DM, Mendolia-Loffredo S, Smith TL. Outcomes of endoscopic sinus surgery for chronic rhinosinusitis associated with sinonasal polyposis. Am J Rhinol 2007;21:84-88. [PubMed: 17283567]

27. Mace J, Yvonne ML, Carlson NE, Litvack JR, Smith TL. Effects of depression on quality of life improvement after endoscopic sinus surgery. Laryngoscope 2008;118:528-534. [PubMed: 18043488]

28. Litvack JR, Griest S, James KE, Smith TL. Endoscopic and quality of life outcomes after revision endoscopic sinus surgery. Laryngoscope 2007;117:2233-2238. [PubMed: 17891047]

29. Soler ZM, Mace J, Smith TL. Fibromyalgia and chronic rhinosinusitis: outcomes after endoscopic sinus surgery. Am J Rhinol 2008;22:427-432. [PubMed: 18702911]

30. Robinson JL, Griest S, James KE, Smith TL. Impact of aspirin intolerance on outcomes of sinus surgery. Laryngoscope 2007;117:825-830. [PubMed: 17473677]

31. Colclasure JC, Gross CW, Kountakis SE. Endoscopic sinus surgery in patients older than sixty. Otolaryngol Head Neck Surg 2004;131:946-949. [PubMed: 15577795]

32. Reh DD, Mace J, Robinson JL, Smith TL. Impact of age on presentation of chronic rhinosinusitis and outcomes after endoscopic sinus surgery. Am J Rhinol 2007;21:207-213. [PubMed: 17424882]

33. Smith TL, Mendolia-Loffredo S, Loehrl TA, Sparapani R, Laud PW, Nattinger AB. Predictive factors and outcomes in endoscopic sinus surgery for chronic rhinosinusitis. Laryngoscope 2005;115:1-7.

34. Soler ZM, Sauer DA, Mace J, Smith TL. Impact of mucosal eosinophilia and nasal polyposis on quality of life outcomes after sinus surgery. Otolaryngol Head Neck Surg 2010;142(1):64-71. [PubMed: 20096225]

35. Ragab SM, Lung VJ, Scadding G. Evaluation of the medical and surgical treatment of chronic rhinosinusitis: a prospective, radomised, controlled trial. Laryngoscope 2004;114:923-930. [PubMed: 15126758]

36. Hartog B, van Benthem PP, Prins LC, Hordijk GJ. Efficacy of sinus irrigation versus sinus irrigation followed by functional endoscopic sinus surgery. Ann Otol Rhinol Laryngol 1997;106:759-766. [PubMed: 9302908]

37. Khalil H, Nunez DA. Functional endoscopic sinus surgery for chronic rhinosinusitis. Cochrane Database of Systematic Reviews. 2006;(Issue 3) Art. No.: CD004458. DOI: 10.1002/14651858.CD004458.pub2. 
Table 1

Minimal clinically important difference for chronic rhinosinusitis quality of life survey instruments

\begin{tabular}{lcc}
\hline $\begin{array}{l}\text { QoL Survey instrument } \\
\text { Domains }\end{array}$ & [score range] & MCID \\
\hline RSDI total & {$[0-120]$} & $\geq 10.35$ \\
Physical & {$[0-44]$} & $\geq 3.80$ \\
Functional & {$[0-36]$} & $\geq 3.45$ \\
Emotional & {$[0-40]$} & $\geq 4.20$ \\
CSS total & {$[0-100]$} & $\geq 9.75$ \\
$\quad$ Symptoms & {$[0-100]$} & $\geq 13.25$ \\
$\quad$ Medications & {$[0-100]$} & $\geq 12.60$ \\
SNOT-22 & {$[0-110]$} & $\geq 8.90$ \\
RQLQ & {$[0-6]$} & $\geq 0.62$ \\
\hline
\end{tabular}

$\mathrm{QOL}=$ quality of life, $\mathrm{MCID}=$ minimal clinically important difference, $\mathrm{RSDI}=$ Rhinosinusitis Disability Index, CSS $=$ Chronic Sinusitis Survey, SNOT-22 = 22 Item Sinonasal Outcomes Test, RQLQ = Rhinitis Quality of Life Questionnaire 
Table 2

Individual symptom scores before and after endoscopic sinus surgery in a cohort of 152 patients with chronic rhinosinusitis

\begin{tabular}{lcccc}
\hline Symptoms: $(\mathbf{n}=152)$ & {$[$ range $]$} & $\begin{array}{c}\text { Preop } \\
\text { mean } \pm \text { SD }\end{array}$ & $\begin{array}{c}\text { Postop } \\
\text { mean } \pm \text { SD }\end{array}$ & p-value \\
\hline Nasal discharge & {$[0-10]$} & $5.49 \pm 3.06$ & $3.38 \pm 2.75$ & $<0.001$ \\
Nasal congestion & {$[0-10]$} & $6.52 \pm 2.84$ & $3.36 \pm 2.79$ & $<0.001$ \\
Facial pain & {$[0-10]$} & $5.45 \pm 2.91$ & $2.34 \pm 2.56$ & $<0.001$ \\
Decreased olfaction & {$[0-10]$} & $5.54 \pm 3.58$ & $2.57 \pm 3.06$ & $<0.001$ \\
Headache & {$[0-10]$} & $4.13 \pm 3.22$ & $4.01 \pm 3.27$ & 0.699 \\
Fatigue & {$[0-10]$} & $6.03 \pm 3.01$ & $3.24 \pm 2.80$ & $<0.001$ \\
Toothache & {$[0-10]$} & $3.32 \pm 3.25$ & $1.17 \pm 2.08$ & $<0.001$ \\
Sinus congestion & {$[0-10]$} & $7.16 \pm 2.46$ & $3.37 \pm 2.85$ & $<0.001$ \\
\hline
\end{tabular}

$\mathrm{SD}=$ standard deviation, $\mathrm{p}<0.05$ = significant. Soler ZM, Mace J, Smith TL. Symptom-based presentation of chronic rhinosinusitis and symptomspecific outcomes after endoscopic sinus surgery. Am J Rhinol 2008: 22:297-301 